\title{
Performance of ESPRIT in the presence of gain and phase mismatches in sensor doublets
}

\author{
G VIJAYA RAGHAVAN ${ }^{1}$, Y YOGANANDAM ${ }^{2}$, \\ Y R K MURALIDHAR ${ }^{3}$ and RAJASEKHAR RAMAN ${ }^{4}$ \\ R and T Unit for Navigational Electronics, Osmania University, Hyderabad \\ 500007 , India \\ Present address: \\ ${ }^{1}$ Department of Electrical Engineering, University of Connecticut, Storrs, \\ CT 06268, USA \\ ${ }^{2}$ Department of Electrical Engineering, Concordia University, 1455 de \\ Maisonneuve West, Montreal H3G IM8, Canada \\ ${ }^{3}$ Department of Electrical Engineering, Oakland University, Rochester, \\ MI 48309-4401, USA \\ ${ }^{4}$ Department of Electrical Engineering, University of Villanova, Villanova, \\ PA 19085, USA
}

\begin{abstract}
ESPRIT, an acronym for Estimation of Signal Parameters via Rotational Invariance Technique, is a novel method for estimating the Directions of Arrival (DOA) of plane waves using an arbitrary array of sensor doublets. However, ESPRIT requires an identical pair of sensors in each doublet, that is the gain and phase characteristics of the sensors in a doublet have to be matched, which may be difficult to ensure in practical situations. In this paper, assuming the sources to be uncorrelated, we analyse the performance of ESPRIT when the gain and phase characteristics of the sensors in a doublet are not identical. It is shown that the angle estimates are unbiased and expressions are derived for the variance in the estimates of DOA, when gain and phase mismatches exist in doublets. Computer simulation results are also presented to assert the theoretical predictions.
\end{abstract}

Keywords. Array signal processing; sensor mismatches; ESPRIT.

\section{Introduction}

Estimation of Directions of Arrival (DOA) of plane waves using an array of sensors has been the subject of active research over many years. Many techniques have been proposed, some non-eigenstructure methods (Reddy et al 1986) and some eigenstructurebased methods (Johnson et al 1982). Recently, Paulraj et al (1986) have proposed ESPRIT, which utilizes the signals received from an arbitrary planar array of sensor doublets. The technique is as follows. 
Consider the array as being made up of two identical subarrays, $X$ and $Y$, displaced doublets whose elements are translationally separated by a known constant displacement vector $\Delta$. The sensor characteristics such as gain and phase patterns, polarization sensitivity etc. may be arbitrary for each doublet as long as the elements are pairwise matched. Assume that there are $D<M$ narrowband sources centred at frequency $\omega$ and located sufficiently far from the array such that the plane wave assumption is valid. Additive noise is present at all $2 M$ sensors and is assumed to be a stationary zero-mean random process that is uncorrelated from sensor to sensor.

Consider the array as being made up of two identical subarrays, $X$ and $Y$, displaced from each other by a known displacement vector $\Delta$. The signals received at the $m$ th doublet can be expressed as

$$
\begin{aligned}
& x_{m}(t)=\sum_{i=1}^{D} a_{m}\left(\theta_{i}\right) s_{i}(t)+n_{x m}(t), \\
& y_{m}(t)=\sum_{i=1}^{D} a_{m}\left(\theta_{i}\right) s_{i}(t) \exp \left(j \beta \sin \theta_{i}\right)+n_{y m}(t),
\end{aligned}
$$

where $s_{i}(\cdot)$ is the $i$ th signal as received at the sensor 1 of the $X$ subarray, $\theta_{i}$ is the DOA of the $i$ th source relative to the broad side direction of $\Delta, a_{m}\left(\theta_{i}\right)$ is the response of the $m$ th sensor of either subarray relative to the response of sensor 1 of the same subarray when a signal wavefront impinges at an angle $\theta_{i}, \beta$ is the wave number $(\beta=(2 \pi \Delta / \lambda))$ corresponding to the centre frequency of operation, and $n_{x m}(\cdot)$ and $n_{y m}(\cdot)$ are the zero mean additive noises at the elements in the $m$ th doublet for subarrays $X$ and $Y$ respectively.

Combining the outputs of each of the sensors in the subarrays, the received data vectors can be written as,

where

$$
\begin{aligned}
& \mathbf{x}(t)=\mathbf{A s}(t)+\mathbf{n}_{x}(t), \\
& \mathbf{y}(t)=\mathbf{A} \Phi \mathbf{s}(t)+\mathbf{n}_{y}(t),
\end{aligned}
$$

$$
\mathbf{x}^{T}(t)=\left[x_{1}(t), x_{2}(t), \ldots, x_{M}(t)\right]
$$

and $\mathbf{y}^{T}(t), \mathbf{n}_{x}^{T}(t)$ and $\mathbf{n}_{y}^{T}(t)$ are similarly defined. The vector $\mathbf{s}(t)$ is a $D$-vector of impinging signals as observed at the reference sensor of the $X$ subarray. The $M \times D$ matrix $A$ is the direction matrix whose columns $\mathbf{a}\left(\theta_{i}\right), i=1, \ldots, D$ are the signal direction vectors for the $D$ wavefronts. The matrix $\Phi$ is a $D \times D$ diagonal matrix of the phase delays $\left(\phi_{i}=\exp \left(j \beta \sin \theta_{i}\right)\right.$ between the doublet sensors for the signals. To estimate the DOA $\left(\theta_{i}\right)$, we form the following auto and cross covariances.

with

$$
\begin{aligned}
& \mathbf{R}_{x x}=E\left[\mathbf{x}(t) \mathbf{x}^{H}(t)\right]=\mathbf{A S} \mathbf{A}^{H}+\sigma^{2} \mathbf{I}, \\
& \mathbf{R}_{x y}=E\left[\mathbf{x}(t) \mathbf{y}^{H}(t)\right]=\mathbf{A S} \Phi^{H} \mathbf{A}^{H},
\end{aligned}
$$

$$
\mathbf{S}=E\left[\mathbf{s}(t) \mathbf{s}^{H}(t)\right]
$$

where $(\cdot)^{H}$ denotes complex conjugate transposition, $E(\cdot)$ is the expectation operation and $\sigma^{2}$ is the variance of the noise. Let

$$
\begin{aligned}
& \mathbf{C}_{x x}=\mathbf{R}_{x x}-\sigma^{2} \mathbf{I}, \\
& \mathbf{C}_{x y}=\mathbf{R}_{x y} .
\end{aligned}
$$


It has been shown by Paulraj et al (1986) that the elements of $\Phi$ are related to the generalized eigenvalues (GEV) of the matrix pair $\left(\mathbf{C}_{x x}, \mathbf{C}_{x y}\right)$ and hence the DOA of the signals can be obtained from these GEV. That is, if $\left(\gamma_{i}\right)$ are the desired generalized eigenvalues close to the unit circle, then the DOA are obtained as

$$
\theta_{i}=\arcsin \left[\left\{\arctan \left[\mathfrak{I}\left(\gamma_{i}\right) / \mathfrak{R}\left(\gamma_{i}\right)\right]\right\} / \beta\right],
$$

where $\mathfrak{R}(\cdot)$ and $\mathfrak{I}(\cdot)$ indicate the real and imaginary parts of a complex quantity. This, however, is possible only when the gain and phase characteristics of the elements in the doublet are identical, which may be difficult to ensure in practice. Any deviation in gain and phase characteristics can cause errors in the estimates of DOA.

In this paper, assuming the sources to be uncorrelated, we analyse the performance of the ESPRIT algorithm in the presence of gain and phase mismatches in the sensor doublets. It is shown that the angle estimates are unbiased. Expressions are derived for the variance in the angle estimates. This is presented in \$2. Computer simulations were performed to assert the predictions made in respect of bias and variance in the angle estimates and the results are presented in $\S 3$. Finally in $\$ 4$, some concluding remarks are presented.

\section{Effect of gain and phase perturbations}

In this section, to study the effect of gain and phase mismatches, we consider the method proposed by Zoltowski (1987) for computing the GEV of a matrix pair.

\subsection{Zoltowski's method}

Consider (5a). Since $\mathrm{C}_{x x}$ is an $M \times M$ hermitian matrix of rank $D$, we can express $\mathrm{C}_{x x}$ as

$$
\mathbf{C}_{x x}=\sum_{i=1}^{D} \alpha_{i} \mathbf{e}_{i} \mathbf{e}_{i}^{H}
$$

where $\left(\alpha_{i}\right)$ and $\left(e_{i}\right)$ are the eigenvalues and the associated eigenvectors of $\mathbf{C}_{x x}$, respectively. Further, since $\mathbf{C}_{x x}$ is a positive semidefinite matrix, it can be decomposed as

where

$$
\mathbf{C}_{x x}=\mathbf{J J}^{H}
$$

$$
J=\left[\sqrt{ } \alpha_{1} \mathbf{e}_{1}, \sqrt{ } \alpha_{2} \mathbf{e}_{2}, \ldots, \sqrt{ } \alpha_{D} \mathbf{e}_{D}\right]
$$

The matrix $\mathbf{J}$ has the following properties:

(i) $\mathbf{J}^{\dagger}=\left[\mathbf{e}_{1}^{*} / \sqrt{ } \alpha_{1}, \mathbf{e}_{2}^{*} / \sqrt{ } \alpha_{2}, \ldots, \mathbf{e}_{D}^{*} / \sqrt{ } \alpha_{D}\right]^{T}$, where ( $\dagger$ ) denotes pseudo-inversion

(ii) $\mathbf{J}^{\dagger} \mathbf{J}=\mathbf{J}^{H}\left(\mathbf{J}^{H}\right)^{\dagger}=\mathbf{I}$

(iii) $\mathbf{J} J^{\dagger}=\left(\mathbf{J}^{H}\right)^{\dagger} \mathbf{J}^{H}=\mathbf{E E} E^{H}=\mathbf{P}$, with $\mathbf{E}=\left[\mathbf{e}_{1}, \mathbf{e}_{2}, \ldots, \mathbf{e}_{D}\right]$ and $\mathbf{P}$ is a projection matrix.

From the properties of the projection matrix, we obtain the following results for further use. Consider the relations in (5). From the structure of $\mathbf{C}_{x x}$ and $\mathbf{C}_{x y}$, we note that the columns of $\mathbf{C}_{x x}$ and $\mathbf{C}_{x y}$ span the same space, defined by the columns of $\mathbf{E}$. Then, for any column vector $\left(\mathbf{c}_{i}, i=1, \ldots, D\right)$ of $\mathbf{C}_{x y}, P \mathbf{c}_{i}=\mathbf{c}_{i}$, which implies that

$$
\mathbf{P C}_{x y}=\mathbf{C}_{x y} \text {. }
$$


Further, consider the product $\mathbf{C}_{x y} \mathbf{P}=\mathbf{Z}$. Post multiplying both the sides by $\mathbf{C}_{x y}$ and collecting the terms to one side yields

$$
\left(C_{x y}-Z\right) C_{x y}=0 .
$$

Since $\mathbf{C}_{x y}$ is not a null matrix, (11) is possible only when $\mathbf{C}_{x y}=\mathbf{A}$, or

$$
\mathbf{C}_{x y} \mathbf{P}=\mathbf{C}_{x y} .
$$

Now consider the solution for the GEV of the matrix pair $\left(\mathbf{C}_{x x}, \mathbf{C}_{x y}\right)$. Let $\gamma_{i}$ and $z_{i}$ be the GEV and GE vectors of the matrix pair. Let

$$
\mathbf{T}=\mathbf{C}_{x x}-\gamma_{i} \mathbf{C}_{x y}
$$

Replacing $\mathbf{C}_{x y}$ in the above equation with $\mathbf{P C}_{x y} \mathbf{P}$ (cf. (10) and (12)) and using (8) for $\mathrm{C}_{x x}$ we get

where

$$
\mathbf{T}=\left[\gamma_{i}^{*} \mathbf{J}\left(\gamma_{i} \mathbf{I}-\mathbf{Q}\right) \mathbf{J}^{H}\right]
$$

$$
\mathbf{Q}=\mathbf{J}^{\dagger} \mathbf{C}_{x y}\left(\mathbf{J}^{H}\right)^{\dagger}
$$

If $\gamma_{i}$ are the GEV of the pair $\left(\mathbf{C}_{x x}, \mathbf{C}_{x y}\right)$, the rank of $\left(\mathbf{C}_{x x}-\gamma_{i} \mathbf{C}_{x y}\right)$ goes below $D$. Since $\operatorname{rank}(\mathbf{J})=\operatorname{rank}\left(\mathbf{J}^{H}\right)=D$, we can see from (14) that the only way it can happen is for the rank of $\left(\gamma_{i}^{*} \mathbf{I}-\mathbf{Q}\right)$ to go below $D$. This implies that $\gamma_{i}^{*}$ form the conventional eigenvalues of $\mathbf{Q}$.

\subsection{Gain and phase mismatches in doublets}

Let the deviation of gain and phase of the $Y$ array sensor from those of the $X$ array sensor in the $m$ th doublet be $\delta a_{m}$ and $\delta \phi_{m}$, respectively, and assume that these errors are independent from doublet to doublet. Then (2), in the presence of gain and phase mismatches, can be written as

$$
\begin{aligned}
& \hat{\mathbf{x}}(t)=\mathbf{A s}(t)+\mathbf{n}_{x}(t) \\
& \hat{\mathbf{y}}(t)=\mathbf{G A} \Phi \mathbf{s}(t)+\mathbf{G n}_{y}(t)
\end{aligned}
$$

where $\mathbf{G}$ is a diagonal matrix of size $M \times M$ with elements

$$
g_{m m}=\left(1+\delta a_{m}\right) \exp \left(j \delta \phi_{m}\right)
$$

Note that in (17), a nominal gain of unity and a nominal phase of zero for the sensors is assumed for the unperturbed array. We also assume that both $\delta a_{m}$ and $\delta \phi_{m}$ are small and zero mean random variables. With this assumption (17) can be simplified to

$$
g_{m m}=1+\delta a_{m}+j \delta \phi_{m}
$$

Using (18), $\mathbf{G}$ can be expressed as

$$
\mathbf{G}=\mathbf{I}+\Delta \mathbf{G}
$$

where $\Delta \mathbf{G}$ is a diagonal matrix with $\left(\delta a_{m}+j \delta \phi_{m}\right)$ as its elements. The perturbed 
covariances $\hat{\mathbf{C}}_{x x}$ and $\hat{\mathbf{C}}_{x y}$ can be obtained as

$$
\begin{aligned}
& \hat{\mathbf{C}}_{x x}=\mathbf{C}_{x x}, \\
& \hat{\mathbf{C}}_{x y}=\mathbf{C}_{x y}+\Delta \mathbf{C}_{x y},
\end{aligned}
$$

where

$$
\Delta \mathbf{C}_{x y}=\mathbf{C}_{x y}(\Delta \mathbf{G})^{H} .
$$

We note from (5) and (20) that the mismatches in the sensors have resulted in the modification of the matrix $\mathbf{C}_{x y}$ by an extra term $\Delta \mathbf{C}_{x y}$. To see the effect of this extra term on the eigenvalues of the matrix $\mathbf{Q}$ of (15) and in turn on the DOA of the sources, we go through the following analysis.

The perturbed $\mathbf{Q}$ matrix, $\hat{\mathbf{Q}}$ is given by

where

$$
\hat{\mathbf{Q}}=\mathbf{J}^{\dagger} \hat{\mathbf{C}}_{x y}\left(\mathbf{J}^{H}\right)^{\dagger}=\mathbf{Q}+\Delta \mathbf{Q}
$$

$$
\Delta \mathbf{Q}=\mathbf{J}^{\dagger} \Delta \mathbf{C}_{x y}\left(\mathbf{J}^{H}\right)^{\dagger}
$$

To see the effect of $\Delta Q$ on the eigenvalues of $Q$, we use the results from Pease (1965, pp. 247-249) as follows.

Consider a matrix $\mathbf{W}$ whose eigenvalues and eigenvectors are given by $\left(\lambda_{i}, \mathbf{u}_{i}\right)$. When $\mathbf{W}$ is perturbed by a matrix $B$ such that $\mathbf{C}=\mathbf{W}+\mathbf{B}$, the eigenvalues and eigenvectors of $\mathbf{C}$ are given by

$$
\begin{gathered}
\tilde{\lambda}_{i}=\lambda_{i}+\eta_{i}, \\
\tilde{\mathbf{u}}_{i}=\mathbf{u}_{i}+\mathbf{v}_{i} .
\end{gathered}
$$

If $\mathbf{W}$ is semisimple i.e., if $\mathbf{W}$ has complete set of linearly independent eigenvectors, $\eta_{i}$ to a first order approximation is given by

$$
\eta_{i}=\mathbf{u}_{i}^{H} \mathbf{K B} \mathbf{u}_{i}
$$

where $\mathbf{K}$ is the metric of the system matrix $\mathbf{W}$ such that

$$
\mathbf{W K}^{-1} \mathbf{W}^{H} \mathbf{K}=\mathbf{K}^{-1} \mathbf{W}^{H} \mathbf{K W} \text {. }
$$

Further, if $\mathbf{W}$ is also unitary, $\mathbf{K}$ will be an identity matrix.

Now consider the matrix $\mathbf{Q}$ given in (15) and $\hat{\mathbf{Q}}$ of (22). From the results in appendix A we note that $\mathbf{Q}$ is unitary and hence semi simple. Therefore the results given in (24) and (25) can directly be applied with $\mathbf{W}=\mathbf{Q}$ and $\mathbf{B}=\Delta \mathbf{Q}$, to give

$$
\eta_{i}=\mathbf{u}_{i}^{H} \Delta \mathbf{Q} \mathbf{u}_{i}
$$

where $\left(\eta_{i}\right)$ are the perturbations in the eigenvalues of matrix $\mathbf{Q}$ and $\left(u_{i}\right)$ are the eigenvectors of $\mathbf{Q}$. Since the eigenvalues of $\mathbf{Q}$ are the complex conjugates of GEV of the pair $\left(\mathbf{C}_{x x}, \mathbf{C}_{x y}\right)$, we can obtain the mean and the mean squared error (MSE) of the perturbation in the GEV as

$$
\begin{gathered}
\mu_{i}=E\left[\eta_{i}\right]=\mathbf{u}_{i}^{H} E[\Delta \mathbf{Q}] \mathbf{u}_{i}, \\
(\mathrm{MSE})_{i}=E\left[\eta_{i} \eta_{i}^{*}\right]=E\left[\mathbf{u}_{i}^{H} \Delta \mathbf{Q} \mathbf{u}_{i} \mathbf{u}_{i}^{H}(\Delta \mathbf{Q})^{H} \mathbf{u}_{i}\right] .
\end{gathered}
$$

Substituting for $\Delta \mathbf{Q}$ from (23) together with (21) in (28) and recalling that the gain 
and phase deviations in the sensors are assumed to be zero mean, it can be shown that

$$
\mu_{i}=0
$$

implying that the GEV of $\left(\mathbf{C}_{x x}, \mathbf{C}_{x y}\right)$ are unbiased and

where

$$
(\mathrm{MSE})_{i}=\mathbf{u}_{i}^{H} \mathbf{J}^{\dagger} \mathbf{C}_{x y} E\left[(\Delta \mathrm{G})^{H} \mathrm{~F} \Delta \mathrm{G}\right] \mathbf{C}_{x y}^{H}\left(\mathbf{J}^{H}\right)^{\dagger} \mathbf{u}_{i}
$$

$$
\mathbf{F}=\left(\mathbf{J}^{\boldsymbol{H}}\right)^{\dagger} \mathbf{u}_{i} \mathbf{u}_{i}^{\boldsymbol{H}} \mathbf{J}^{\dagger}
$$

Since $\Delta \mathbf{G}$ is a diagonal matrix, we can simplify (30b) to give

where

$$
(\mathrm{MSE})_{i}=\mathbf{u}_{i}^{H} \mathbf{J}^{\dagger} \mathbf{C}_{x y} \tilde{\mathbf{F}} \mathbf{C}_{x y}\left(\mathbf{J}^{H}\right)^{\dagger} \mathbf{u}_{i},
$$

with

$$
\tilde{\mathbf{F}}=\operatorname{diag}\left[\sigma_{g_{1}}^{2} f_{11}, \cdots, \sigma_{\theta_{M}}^{2} f_{M M}\right] \text {, }
$$

$$
\sigma_{g m}^{2}=\sigma_{a m}^{2}+\sigma_{p m}^{2}
$$

and $f_{i j}$ is the $i$-jth element of matrix F. In (34) $\sigma_{a m}^{2}$ and $\sigma_{p m}^{2}$ are the variances of the gain and phase perturbations in the $m$ th doublet.

Now, in what follows, we obtain the relations for the bias and variance of the DOA estimates. Let $\Delta \theta_{i}$ be the perturbation in the DOA $\left(\theta_{i}\right)$ due to mismatches in the sensors within the doublets. Then the true and perturbed GEV are related to $\theta_{i}$ and $\Delta \theta_{i}$ as

$$
\begin{aligned}
& \gamma_{i}^{*}=\exp \left[-j \beta \sin \theta_{i}\right], \\
& \hat{\gamma}_{i}^{*}=(1+\Delta r) \exp \left[-j \beta \sin \left(\theta_{i}+\Delta \theta_{i}\right)\right],
\end{aligned}
$$

where $\Delta r$ is the magnitude perturbation in the eigenvalue $\gamma_{i}^{*}$. Let

$$
\tilde{\gamma}_{i}^{*}=\gamma_{i}^{*}+\eta_{i},
$$

where $\eta_{i}$ is the perturbation in the eigenvalues. From the results of appendix B we obtain

$$
\Delta \theta_{i}=\mathfrak{J}\left(\eta_{i} \gamma_{i}^{*}\right) /\left(\beta \cos \theta_{i}\right)
$$

From (37) we can obtain the mean and variance of $\Delta \theta_{i}$ in terms of the GEV as

where

$$
\begin{aligned}
& \mu_{\theta_{i}}=E\left[\Delta \theta_{i}\right]=0, \\
& \sigma_{\theta_{i}}^{2}=E\left[\Delta \theta_{i}^{2}\right]=\left\{2 \sigma_{i}^{2}-\left[\left(\sigma_{n}^{*}\right)^{2} \gamma_{i}^{2}-\sigma_{n}^{2}\left(\gamma_{i}^{*}\right)^{2}\right]\right\} /\left\{4\left(\beta \cos \theta_{i}\right)^{2}\right\},
\end{aligned}
$$

$$
\sigma_{n}^{2}=E\left[\eta_{i}^{2}\right]=\mathbf{u}_{i}^{H} \mathbf{J}^{\dagger} \mathbf{C}_{x y} \mathbf{\Sigma H}\left(\mathbf{J}^{H}\right)^{\dagger} \mathbf{u}_{i}
$$

with $\Sigma$ being the diagonal matrix whose $m$ th element is $\left(\sigma_{a m}^{2}-\sigma_{p m}^{2}\right)$ and

$$
\mathbf{H}=\left(\mathbf{J}^{H}\right)^{\dagger} \mathbf{u}_{l} \mathbf{u}_{i}^{H} \mathbf{J}^{\dagger} \mathbf{C}_{x y}
$$

Thus, in the presence of gain and phase mismatches the angle estimates are unbiased and the variance can be computed using (39) together with (40) and (41).

From (39) we note that the variance in the DOA estimates for a given set of gain and phase perturbations increases as the $\left(\theta_{i}\right)$ move away from the boresight direction 


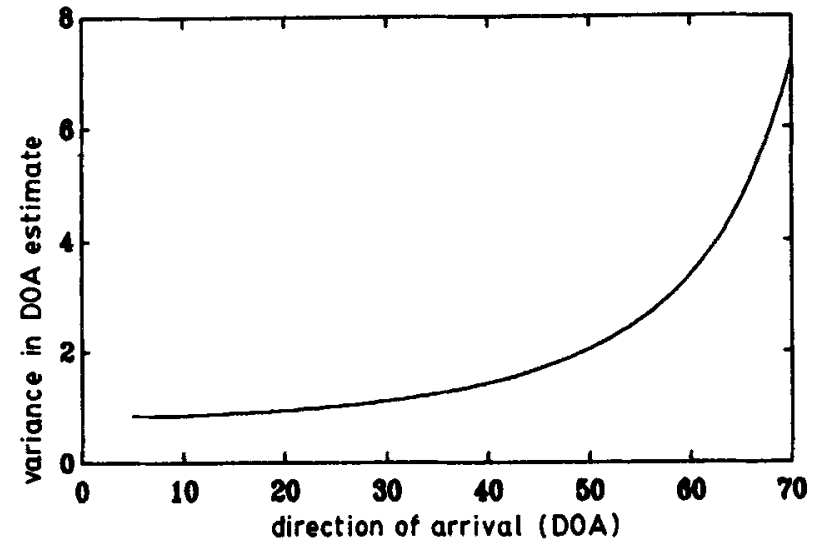

Figure 1. Plot of variance in DOA estimates.

of the displacement vector. To see the nature of this increase in variance, let us consider the case of a single source and a linear array of 4 equispaced doublets with maximum gain and phase perturbation as 0.1 and $10^{\circ}$, respectively. In figure 1 we have plotted the variance in the DOA estimates as a function of DOA.

To see the effect of array size on the variance we shall consider a source with $D O A=10^{\circ}$. In figure 2 we have plotted the variance as a function of the number of doublets whose gain and phase perturbations take the maximum values of 0.1 and $10^{\circ}$ respectively. From this plot we observe that the variance in the DOA estimates decreases as the array size increases. In the next section we present some simulation results to see how well the theoretical expressions predict the variance in the angle estimates.

\section{Simulation results}

In this section we present some simulation results to verify the predictions made in the earlier section in respect of bias and variance in the DOA estimates. The following scenario is assumed for the purpose of simulation. Two sources from $10^{\circ}$ and $20^{\circ}$ with respect to broad side direction of displacement vector impinge on a linear array of four equispaced doublets whose interelement spacing is $\lambda / 2$. The nominal gain and

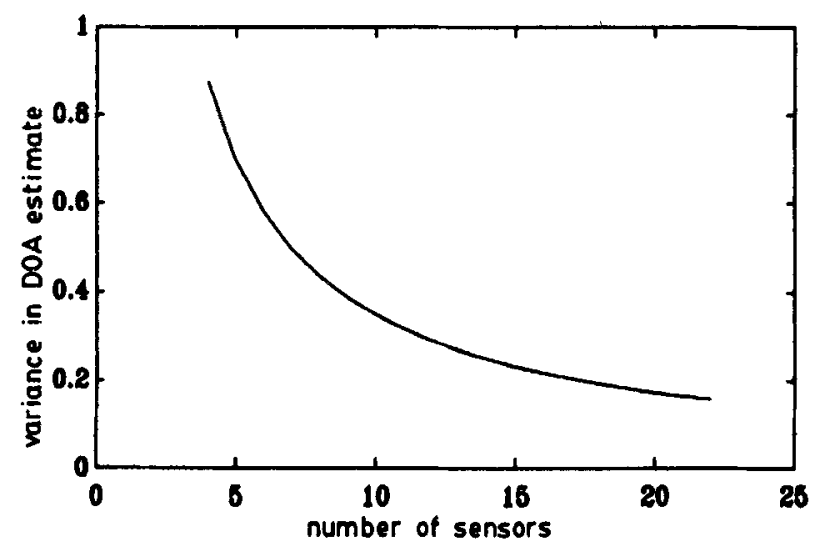

Figure 2. Plot of variance in DOA estimates. 
phase values for the sensors are unity and zero respectively. The gain and phase perturbations of the sensors are assumed to be samples from uniform distribution with $\pm a_{\max }$ and $\pm p_{\max }$ as the limits on the gain and phase perturbations. Since the analysis of the previous section was based on the asymptotic covariance matrices, simulations were carried out for the same.

The perturbed covariance matrices $\hat{\mathbf{C}}_{x x}$ and $\hat{\mathbf{C}}_{x y}$ were formed as

$$
\begin{aligned}
& \hat{\mathbf{C}}_{x x}=\mathbf{A S} \mathbf{A}^{H}, \\
& \hat{\mathbf{C}}_{x y}=\mathbf{A S} \Phi^{H} \mathbf{A}^{H} \mathbf{G}^{H},
\end{aligned}
$$

where the entries of the matrix $\mathbf{G}, g_{m m}$ are as in (17). The perturbation values $\delta a_{m}$ and $\delta \phi_{m}$ are assumed to be the samples of zero mean uniform random processes with $\pm a_{\max }, \pm p_{\max }$ as the limits for amplitude and phase deviation, respectively. To estimate the DOA, the generalized eigenvalues of the pair $\left(\hat{\mathbf{C}}_{x x}, \hat{\mathbf{C}}_{x y}\right)$ were computed as the complex conjugates of the conventional eigenvalues of the matrix $\mathbf{Q}$, given in (15) with $\mathbf{C}_{x y}$ replaced by $\hat{\mathbf{C}}_{x y}$ and the DOA estimated using the inverse relation as in (6). This experiment is repeated for $N$ trials, where $N=500$. The mean and variance in the angle estimates thus obtained are computed as

$$
\mu_{\theta_{i}}=\frac{1}{N} \sum_{i=1}^{N} \hat{\theta}_{i}
$$
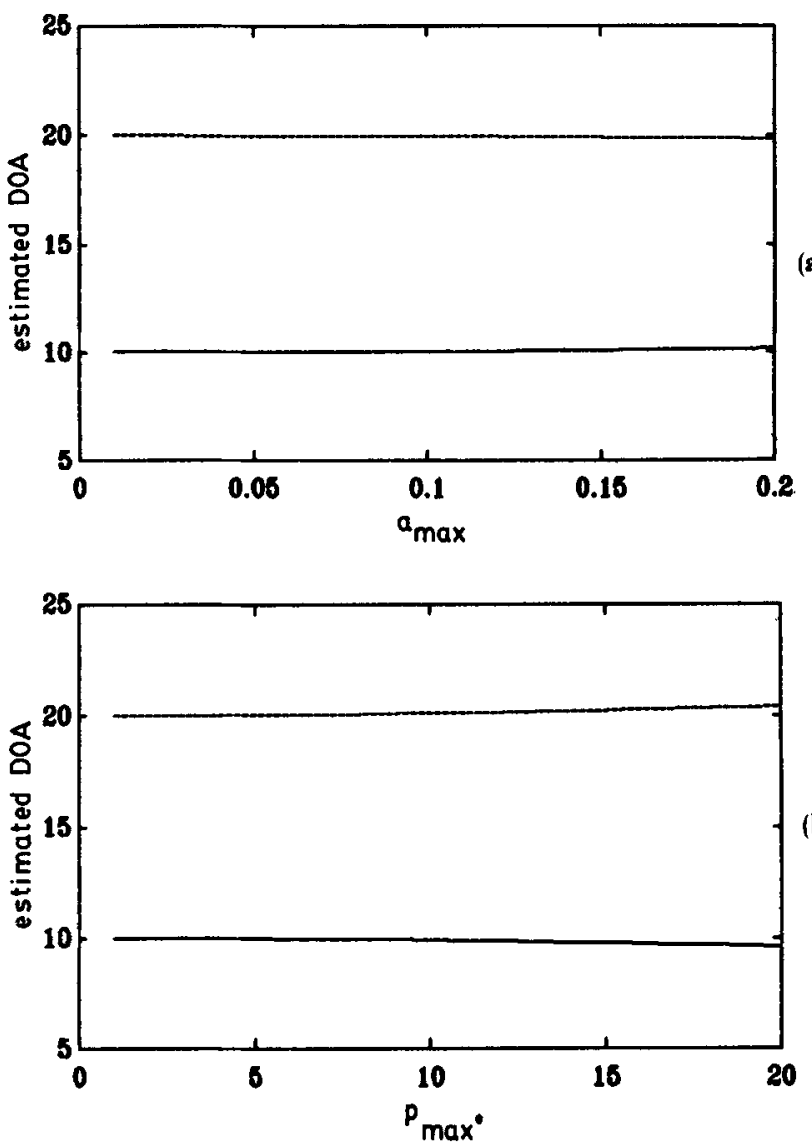

(a)

(b)

Figure 3. Plots of estimated values of DOA as functions of gain perturbation (a), and phase perturbation (b). 


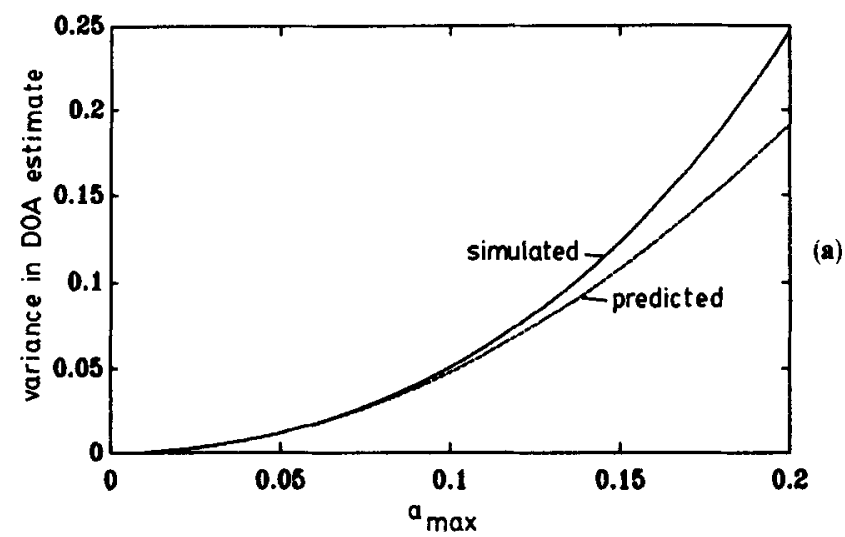

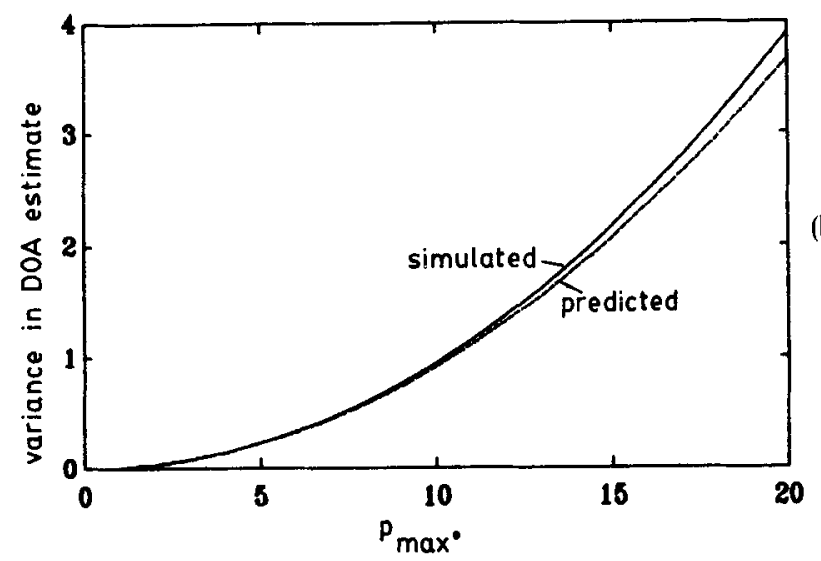

$\sigma_{\theta_{i}}^{2}=\frac{1}{N} \sum_{i=1}^{N}\left(\hat{\theta}_{i}-\mu_{\theta_{i}}\right)^{2}$ (a)

(b)

Figure 4. Plots of variance in DOA estimates $-\mathrm{DOA}=10^{\circ}$.

The above simulations were carried out for various values of $a_{\max }$ and $p_{\max }$. In figures $3 a$ and $b$ we have plotted the mean of the angle estimates as a function of $a_{\max }$ and $p_{\max }$, respectively. One may note here that the mean of the error in DOA estimates should be zero as shown in (39). From figures $3 \mathrm{a}$ and $\mathrm{b}$ we note that the estimated DOA are close to $10^{\circ}$ and $20^{\circ}$ which shows that the estimates are almost unbiased. In figures $4 \mathrm{a}$ and $\mathrm{b}$ we have plotted the variance in the DOA estimates for the case $\theta_{i}=10^{\circ}$, as a function of $a_{\max }$ and $p_{\max }$. In the same figure the theoretically predicted value, computed using (40) is aiso plotted. The results for the case of $\theta_{i}=20^{\circ}$ are plotted in figures $5 \mathrm{a}$ and $\mathrm{b}$. From these plots we conclude that the variance in the DOA estimates is quite small even for a $20 \%$ perturbation in gain over and above the nominal value. Further we also note that the predicted values are in close agreement with those obtained through simulations. To see the combined effect of gain and phase perturbation, some sample results are presented in table 1.

\section{Conclusions}

In this paper we addressed the problem of sensor gain and phase mismatches in doublets used for estimating directions-of-arrival using ESPRIT. It is shown that when 


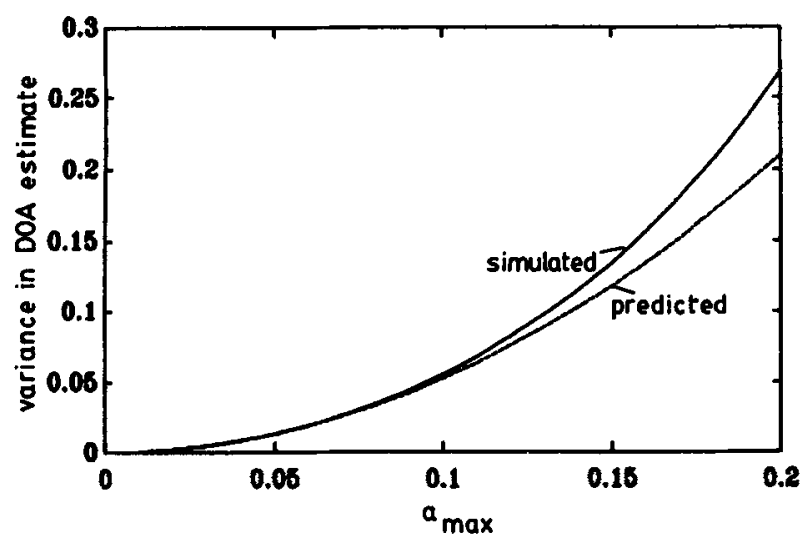

(a)

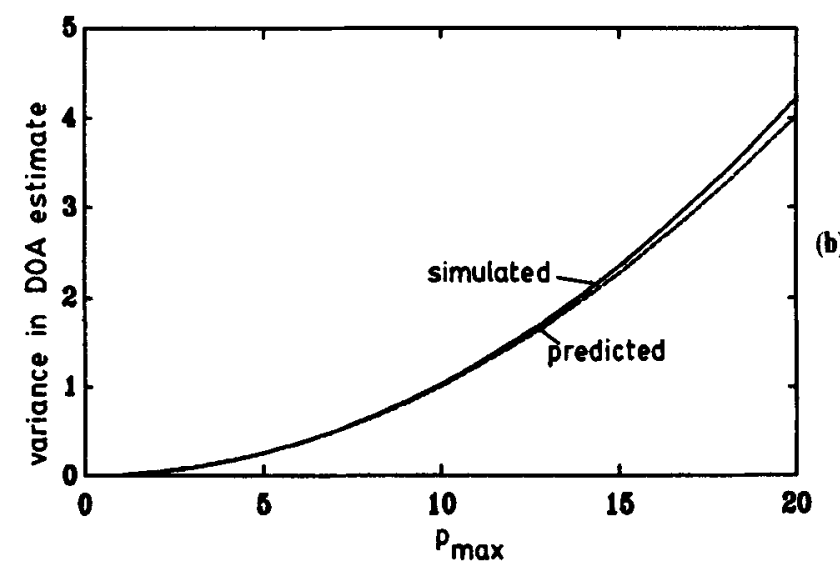

Figure 5. Plots of variance in DOA estimates $-\mathrm{DOA}=20^{\circ}$.

the sources are uncorrelated the angle estimates are unbiased and an expression for the variance in the DOA estimates is derived. Simulation results are also presented which confirm the theoretical predictions.

Table 1. Effect of both gain and phase perturbations.

$D=2, \mathrm{DOA}=10^{\circ}$ and $20^{\circ}, M=4$

\begin{tabular}{|c|c|c|c|c|}
\hline \multirow[b]{2}{*}{$a_{\max }$} & \multirow[b]{2}{*}{$p_{\max }$ (degrees) } & \multirow[b]{2}{*}{ Mean of DOA estimates (degrees) } & \multicolumn{2}{|c|}{ Variance in DOA estimates (degrees) } \\
\hline & & & (Simulation) & (Theoretical) \\
\hline 0.05 & 5 & $\begin{array}{r}9.9823 \\
20.0194\end{array}$ & $\begin{array}{l}0.2370 \\
0.2529\end{array}$ & $\begin{array}{l}0.2408 \\
0.2645\end{array}$ \\
\hline $0 \cdot 10$ & 10 & $\begin{array}{r}9.9301 \\
20.0765\end{array}$ & $\begin{array}{l}0.9500 \\
1.0247\end{array}$ & $\begin{array}{l}0.9632 \\
1.0580\end{array}$ \\
\hline 0.05 & 10 & $\begin{array}{r}9 \cdot 9058 \\
20 \cdot 1026\end{array}$ & $\begin{array}{l}0.9061 \\
0.9929\end{array}$ & $\begin{array}{l}0.92747 \\
1.0186\end{array}$ \\
\hline $0 \cdot 10$ & 5 & $\begin{array}{r}9.9918 \\
20.0079\end{array}$ & $\begin{array}{l}0.2788 \\
0.2897\end{array}$ & $\begin{array}{l}0.2766 \\
0.3038\end{array}$ \\
\hline $0 \cdot 15$ & 15 & $\begin{array}{r}9.8462 \\
20 \cdot 1689\end{array}$ & $\begin{array}{l}2 \cdot 1514 \\
2 \cdot 3463\end{array}$ & $\begin{array}{l}2 \cdot 1673 \\
2 \cdot 3804\end{array}$ \\
\hline
\end{tabular}




\section{Appendix A}

In this appendix we show that the matrix $\mathbf{Q}$, defined in (15) of $\S 2.1$, is unitary and semi simple. Consider

Let

$$
\mathbf{Q}=\mathbf{J}^{\dagger} \mathbf{C}_{x y}\left(\mathbf{J}^{\dagger}\right)^{\boldsymbol{H}}
$$

$$
\mathbf{Z}=\mathbf{Q} \mathbf{Q}^{H}=\mathbf{J}^{\dagger} \mathbf{C}_{x y}\left(\mathbf{J}^{\dagger}\right)^{H} \mathbf{J}^{\dagger}\left(\mathbf{C}_{x y}\right)^{H}\left(\mathbf{J}^{\dagger}\right)^{H}
$$

Consider the product

$$
\left(\mathbf{J}^{\dagger}\right)^{H} \mathbf{J}^{\dagger}=\left(\mathbf{J} \mathbf{J}^{H}\right)^{\dagger}=\mathbf{C}_{x x}^{\dagger}=\left(\mathbf{A S A}{ }^{H}\right)^{\dagger}=\left(\mathbf{A}^{H}\right)^{\dagger} \mathbf{S}^{\dagger} \mathbf{A}^{\dagger}
$$

One may note here that the reversing of pseudoinverses, as used in the above equation, is not always true. However, in this case, it is valid and can be proved easily by expressing $A$ in terms of SVD and using the Moore-Penrose identity ( $\mathbf{X X}^{\dagger} \mathbf{X}=\mathbf{X}$ ). Using (A3) in (A2) and noting that the sources are uncorrelated, we obtain

or

$$
\mathbf{Z}=\mathbf{J}^{\dagger} \mathbf{A S} \Phi^{H} \mathbf{A}^{H}\left(\mathbf{A}^{H}\right)^{\dagger} \mathbf{S}^{\dagger} \mathbf{A}^{\dagger} \mathbf{A S}^{H} \Phi \mathbf{A}^{H}\left(\mathbf{J}^{\dagger}\right)^{H},
$$

$$
\mathbf{Z}=\mathbf{J}^{\dagger} \mathbf{A S} \Phi^{H} \mathbf{S}^{\dagger} \mathbf{S}^{H} \Phi \mathbf{A}^{H}\left(\mathbf{J}^{\dagger}\right)^{H}
$$

Since $\mathbf{S}$ is a real matrix, $\mathbf{S}^{\dagger} \mathbf{S}^{H}=\mathbf{S}^{\dagger} \mathbf{S}=\mathbf{I}$ and $\Phi^{H} \Phi=\mathbf{I}$. Then (A5) reduces to

$$
\mathbf{Z}=\mathbf{J}^{\dagger} \mathbf{A S A}^{H}\left(\mathbf{J}^{\dagger}\right)^{H}=\mathbf{J}^{\dagger} \mathbf{C}_{x x}\left(\mathbf{J}^{\dagger}\right)^{H}=\mathbf{J}^{\dagger} \mathbf{J} \mathbf{J}^{H}\left(\mathbf{J}^{\dagger}\right)^{H}=\mathbf{I}
$$

Similarly we can show that $\mathbf{Q}^{\boldsymbol{H}} \mathbf{Q}=\mathbf{I}$. Hence, $\mathbf{Q}$ is unitary.

Since $\mathbf{Q}$ is a $D \times D$ unitary matrix, with rank $D$, it has a complete set of linearly independent (orthogonal) eigenvectors. A matrix having a complete set of linearly independent eigenvectors is called semi simple. Hence $\mathbf{Q}$ is semi simple.

\section{Appendix B}

In this appendix we relate the perturbation in the DOA to the eigenvalue perturbation of the matrix Q. From (35) of $\$ 2.2$

Let

$$
\begin{aligned}
& \gamma_{i}^{*}=\exp \left[-j \beta \sin \theta_{i}\right] \\
& \hat{\gamma}_{i}^{*}=(1+\Delta r) \exp \left[-\beta \sin \left(\theta_{i}+\Delta \theta_{i}\right)\right]=\gamma_{i}^{*}+\eta_{i}
\end{aligned}
$$

Then

$$
\begin{aligned}
& \gamma_{i}=p+j q \\
& \eta_{i}=\delta p-j \delta q .
\end{aligned}
$$

From (B1)-(B4) we can obtain the following

$$
\begin{aligned}
\beta \sin \theta_{i} & =\arctan (q / p), \\
\beta \sin \left(\theta_{i}+\Delta \theta_{i}\right) & =\arctan [(q+\delta q) /(p+\delta p)] .
\end{aligned}
$$


Assuming $\Delta \theta_{i}$ to be small, we obtain from (B6) and (B7)

$$
\Delta \theta_{i}=1 /\left(\beta \cos \theta_{i}\right) \arctan [(p \delta q-q \delta p) /(l+p \delta p+q \delta q)] .
$$

Using the property that for small arguments $\arctan (\delta a)=\delta a$ and assuming that $(p \delta p+q \delta q)$ is small compared to 1 , we obtain

$$
\Delta \theta_{i}=\left(l / \beta \cos \theta_{i}\right)[p \delta q-q \delta p] .
$$

Expressing $p, q, \delta p, \delta q$ in terms of $\gamma_{i}$ and $\eta_{i}$, we obtain

$$
\Delta \theta_{i}=\mathfrak{I}\left(\eta_{i} \gamma_{i}^{*}\right) /\left(\beta \cos \theta_{i}\right)
$$

\section{References}

Johnson D H, DeGraaf S R 1982 Improving the resolution of bearing in passive sonar arrays by eigenvalue analysis. IEEE Trans. Acoust., Speech Signal Process. ASSP-30: 638-647

Paulraj A, Roy R, Kailath T 1986 A subspace rotation approach to signal parameter estimation. Proc. IEEE 74: 1044-1045

Pease M C III 1965 Methods of matrix algebra (New York, London: Academic Press)

Reddy V U, Yoganandam Y, Rajendra Kumar 1986 AR and eigenstructure methods for estimating direction of arrival of multiple plane waves. J. Inst. Electron. Telecommun. Eng. 32: 361-373

Zoltowiski M D 1987 Solving generalized eigenvalue problem with singular forms. Proc. IEEE 75: 1546-1548 